\title{
Illumination Invariant Object Detection and Tracking with Pre- Equalization and Mean Shift
}

\author{
Adinarayana Ekkurthi ${ }^{1}$, Dr.G.Sudhavani ${ }^{2}$ \\ ${ }^{I}$ Research Scholar, Dept .of ECE, Acharya Nagarjuna University, Guntur, A.P, India. \\ Associate Professor,Dept.of ECE,SMCE,NH-16,Guntur,A.P,India. \\ ${ }^{2}$ Professor, Dept of ECE, R.V.R \& J.C College of Engineering, Guntur, A.P, India.
}

\begin{abstract}
Video surveillance is commonly observed in many places. The video recording is intended to detect and track a particular object or a person One of the major issues related to these video recording is changing of illumination during recording at different intervals of time, which degrades the working performance of detection algorithm. In this paper, a simple and fast pre-equalization approach is proposed along with mean shift tracking. Each frame that is supposed to be processed for detection is normalized and the intensity values are adjusted with the use of histogram properties and then the frame is fed to tracking algorithm. Experimental results shows that the proposed approach is providing promising results when compared with the results obtained under low or varying illumination and other equalization methods.
\end{abstract}

Keywords: Object detection, Object tracking, Equalization, Mean Shift tracking.

\section{Introduction}

Object detection and tracking in the surveillance video is one of the challenging tasks in computer vision research, where the object of interest is supposed for recognition and track its existence in a sequence of frames. This has become the more important topic of interest due to its vast applications in the field of security. Three main steps are involved in this process, to detect the object of interest, trace the movement of the object from frame to frame and analyze its behaviour of movement. This article is mainly focused on detection of an object of interest and tracking its movement in each frame only.

Object detection algorithms often fail to provide maximum performance due to the changing illumination and tracking of object movement becomes critical. Several approaches were discussed so far in the literature to overcome this issue, in [1] Comaniciu et al, proposed a robust and low complexity mean shift tracking algorithm but it doesn't provide satisfactory results when the background illumination is varied. In [2] Lipton et al, a frame difference based moving region extraction using the pixel differences between the consecutive frames. In [3] Liu et.al proposed background subtraction method by taking the difference between current and reference background images. This work fails when the illumination in the reference and the background frames have differed which is practically impossible. In [4] Desa et.al proposed a combination of background subtraction and frame difference, this is an improved version of the work done by Liu but still, suffers from the same limitations. In [4] Celik et.al, introduces an equalized approach to enhance the contrast levels in the frame with Gaussian mixture models, however this cannot enhance the very low illuminated image because the enhancement depends on the histogram of the images or a frame.

To address the above-mentioned limitation a generalized pre-equalization approach is proposed in this paper to enhance the contrast level of the frame before the object of interest has been tracked. This paper is organized as follows; Section I presents the concept of object detection in computer vision and the application of tracking in the current world. Section II presents a brief theoretical and mathematical explanation of the concepts that were involved in the designing of the proposed approach, Section III presents the proposed approach, and section IV presents the experimental results that were obtained with the proposed approach and its validations. Finally, conclusions are heightened in section V.

\section{(A) Pre-Equalization}

\section{Relateded Work}

Consider a color image can be represented as a function of $f=\left(f_{r}, f_{g}, f_{b}\right)$, the available range for each component is [ 0 255], the respective histogram of each component can be represented as $\left\{h_{c}, p_{c}\right\}$ where $c=$ $r, g, b$. Here $h_{c}$ represents total intensity values in the image and $p_{c}$ is the total non-zero probability of all the intensity values. As stated in [5] it can be assumed as 


$$
\left(\frac{\int|f(x)|^{\alpha} d x}{\int d x}\right)^{\frac{1}{\alpha}}=\left(\begin{array}{c}
\left(p_{r}^{T} h_{r}^{T}\right)^{\frac{1}{\alpha}} \\
\left(p_{g}^{T} h_{g}^{T}\right)^{\frac{1}{\alpha}} \\
\left(p_{b}^{T} h_{b}^{T}\right)^{\frac{1}{\alpha}}
\end{array}\right)
$$

Given an image normalized estimation of light source can be estimated as

$$
e_{c}(\alpha)=\frac{\left(p_{c}^{T} \overline{h_{c}^{\alpha}}\right)^{\frac{1}{\alpha}}}{\sqrt{\sum_{c}\left(p_{r}^{T} \overline{h_{r}^{\alpha}}\right)^{\frac{2}{\alpha}}}}
$$

The balanced histogram can be obtained as

$$
\overrightarrow{h_{c}}=\frac{1}{e_{c}(\alpha) \sqrt{3}} \overline{h_{c}}
$$

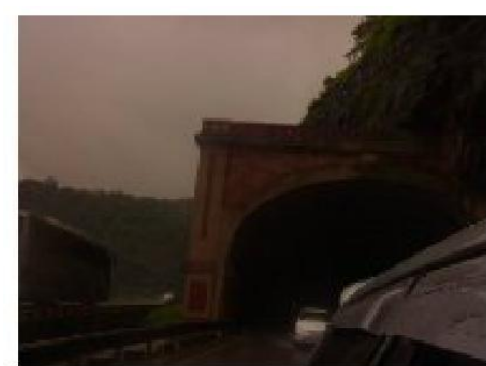

Figure 1: a) Original image

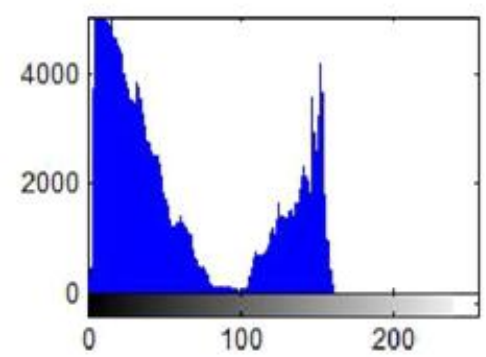

Figure 2: a) Original Image Histogram

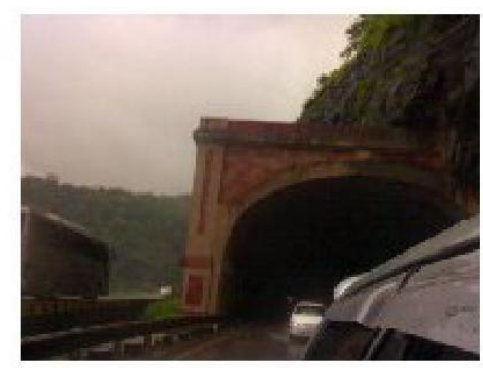

b) Enhanced image

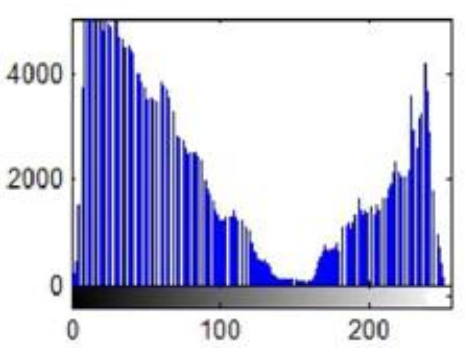

b) Enhanced Image Histogram

The proposed approach can be used for any illumination variation and also can be used to minimize the haze effect introduced by the atmosphere. This makes it more useful to adopt this approach for surveillance videos where apart from illumination variation the video content changes with the intrusion of atmospheric effects like smoke, snow, fog etc.

\section{(B) Mean-Shift Tracking (MST) Algorithm}

This algorithm is proposed by Comaniciu in [1], which is fast and accurate object tracking algorithm. This approach is aimed to find clusters in the joint spatial and color space randomly chosen from the data. Each cluster center is moved to the mean of the data lying inside the multi-dimensional ellipsoid centered in the 
cluster center. The vector defined by the old and the new cluster centers us called the mean shift vector and this process is repeated iteratively until the clusters do not change their positions. In this paper Epanechnikov based kernel is used, the center of the next frame of the target is given as

$$
Y=\frac{\sum_{i=1}^{n} x_{i} w_{i} g\left(\frac{\left(Y_{o}-x_{i}\right)^{2}}{H}\right)}{\sum_{i=1}^{n} w_{i} g\left(\frac{\left(Y_{o}-x_{i}\right)^{2}}{H}\right)}
$$

Where ' $\mathrm{w}$ ' is weight calculated from the target model and target candidate which used to calculate the new center, ' $\mathrm{g}$ ' is the negative of kernel ' $\mathrm{k}$ ', Y0 is the old center. Similarity measurement is calculated with Bhattacharya distance and repeated until the candidate model is close to target model [6].

\section{Proposed Approach}

The surveillance videos often corrupted by the different issues like atmospheric effects (Smoke, snow fog etc), poor illumination, Varying illumination and many. In this section, a pre- equalized approach is proposed for the video with poor and varying illumination. Consider a video sequence in which the illumination is varied differently for different frames an example is presented in below figure

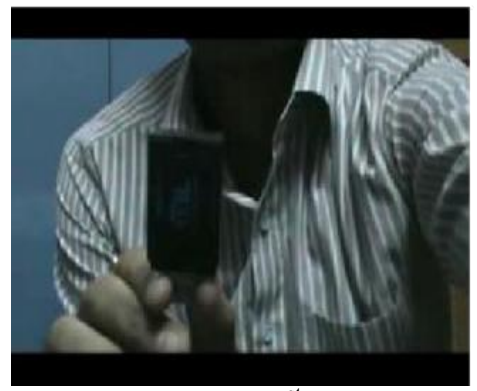

Figure 3: (a) $1^{\text {st }}$ Frame

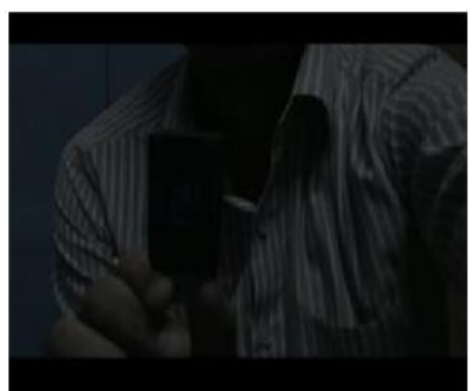

(b) $4^{\text {th }}$ Frame

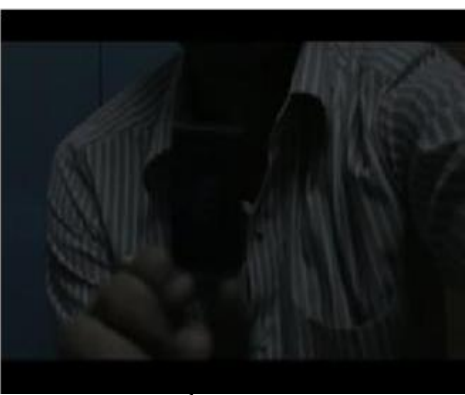

(c) $8^{\text {th }}$ Frame

Each video sequence is processed in a frame by frame manner individually, for each individual frame the preequalization approach is applied to enhance its visual contrast levels using the algorithm stated in section II (A), the resultant enhanced frame is depicted below

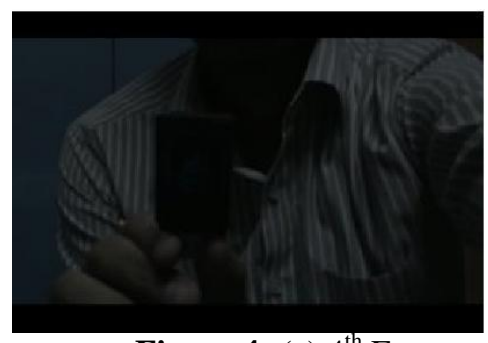

Figure 4: (a) $4^{\text {th }}$ Frame

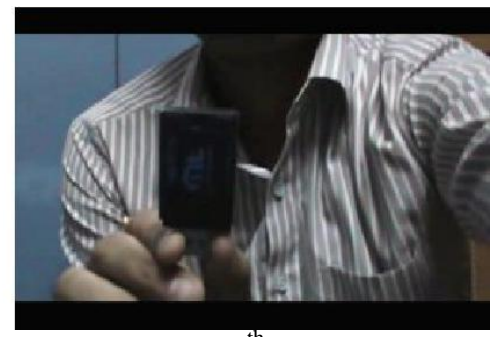

(b) Enhanced $4^{\text {th }}$ Frame

These enhanced frames are fed to mean shift algorithm for the detection of object of interest, the section of the object is provided by the user as shown below

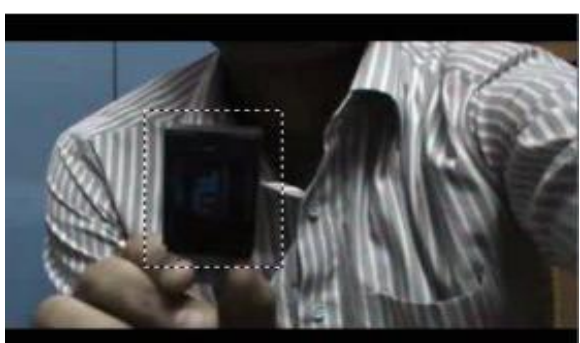

Figure 5: Selection of object of Interest 
The selected objected is marked and treated as a region of interest for which the initial clusters are selected using means shift algorithm. The region is now represented as shown below

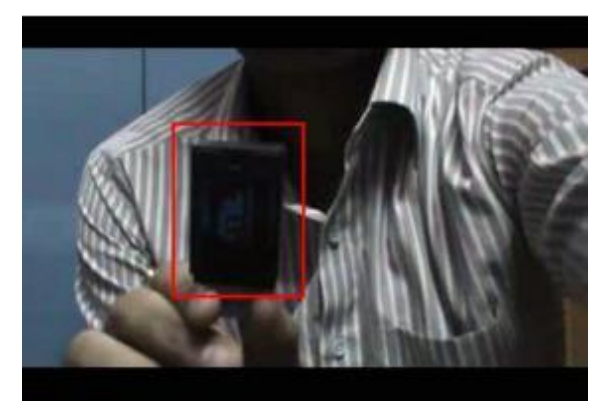

Figure 6: Selected marked region as reference for Region of Interest

\section{Experimental Results}

To evaluate the performance of the proposed approach and to validate the algorithm, the approach is tested with both real-time and standard database videos (Pets 2006 [7]) on Windows 7 OS, 4GB RAM system with Matlab 2013a version. Real time videos are recorded with Dauhua [8] bullet camera and the video resolution is $352 \times 240$, the illumination is varied synthetically. The results obtained with pre-equalization and without are presented below

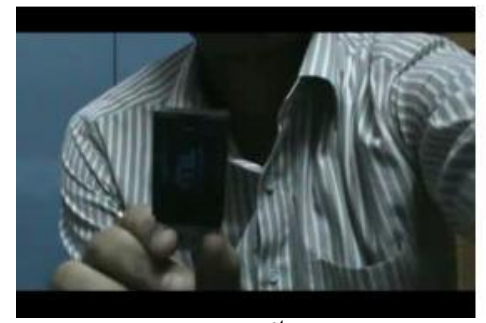

(a) $1^{\text {st }}$ Frame

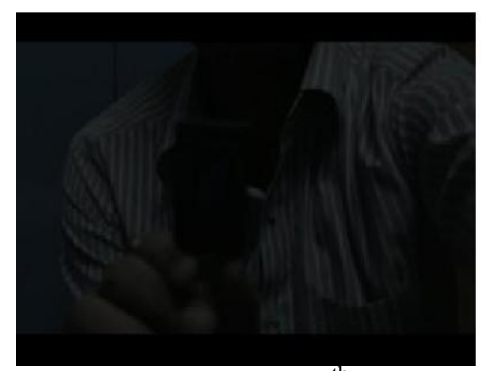

(c) $7^{\text {th }}$ Frame

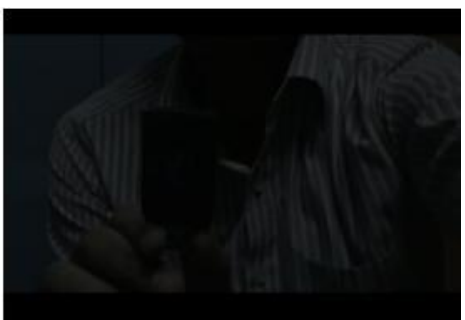

(b) $3^{\text {rd }}$ Frame

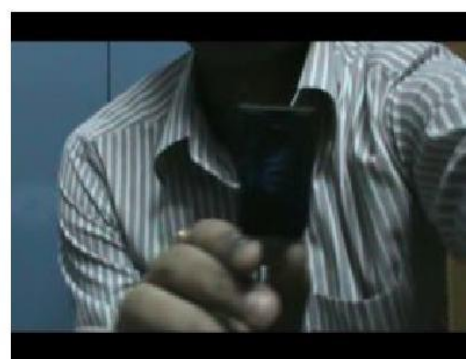

(d) $17^{\text {th }}$ Frame

Figure 7: Different frames from original input video sequence
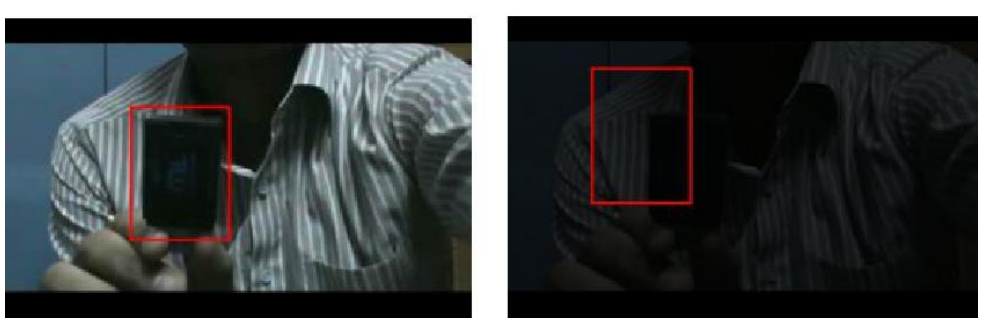

(a) $1^{\text {st }}$ Frame

(b) $3^{\text {rd }}$ Frame
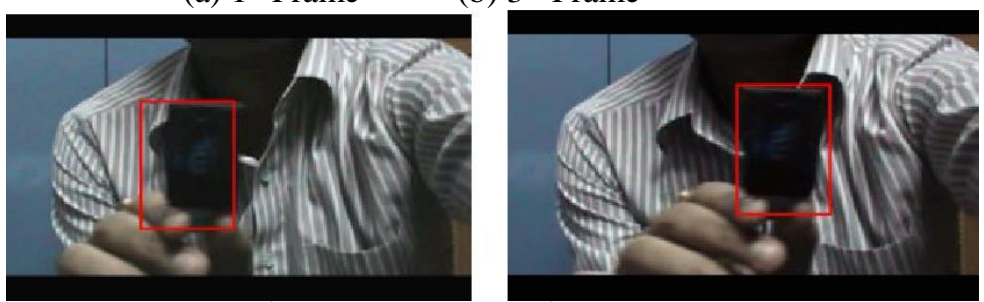

(c) $7^{\text {th }}$ Frame

(d) $17^{\text {th }}$ Frame

Figure 8: Resultant video sequence with conventional Mean-shift tracking 


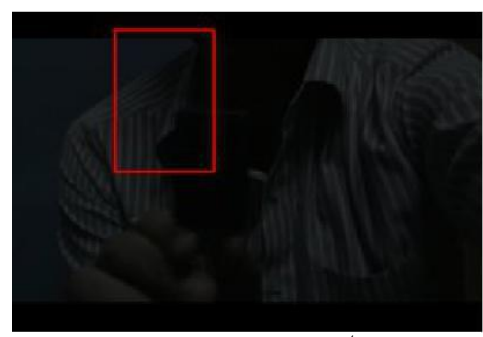

(a) $1^{\text {st }}$ Frame

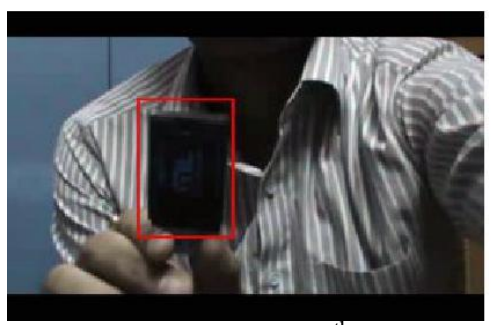

(c) $7^{\text {th }}$ Frame

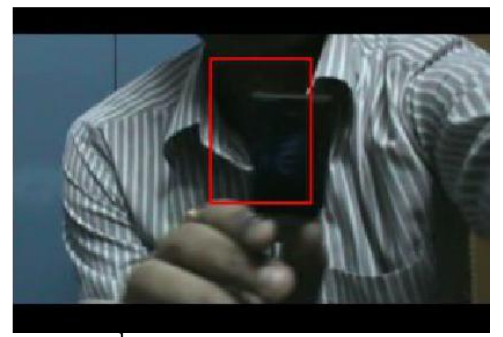

(b) $3^{\text {rd }}$ Frame

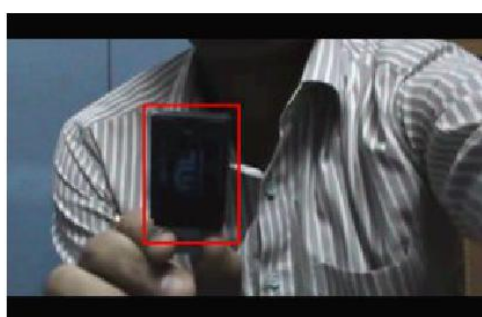

(d) $17^{\text {th }}$ Frame

Figure 9: Resultant video sequence with proposed approach

It is observed from the experimental results that the proposed approach while preserving the originality of the input frame it able to detect the ROI accurately. The results are compared are validate with respect to ground truth (segmented by experts) and the performance is depicted below

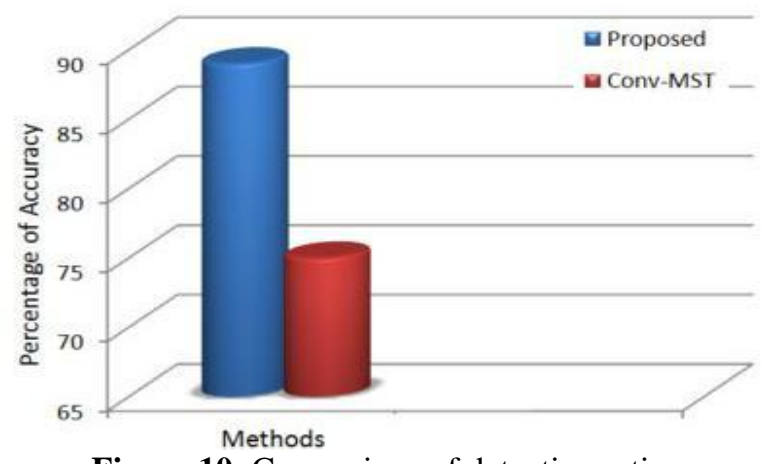

Figure 10: Comparison of detection ratio

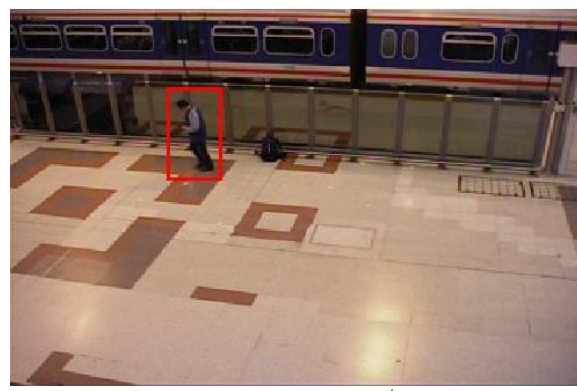

(a) $1^{\text {st }}$ Frame

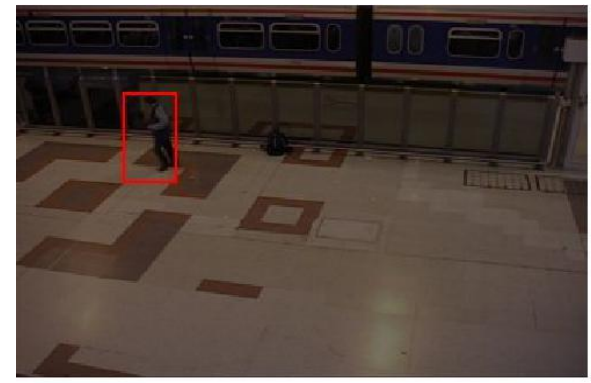

(c) $15^{\text {th }}$ Frame

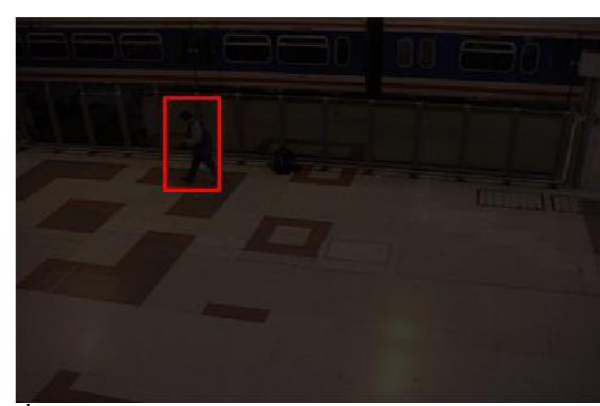

(b) $4^{\text {th }}$ Frame

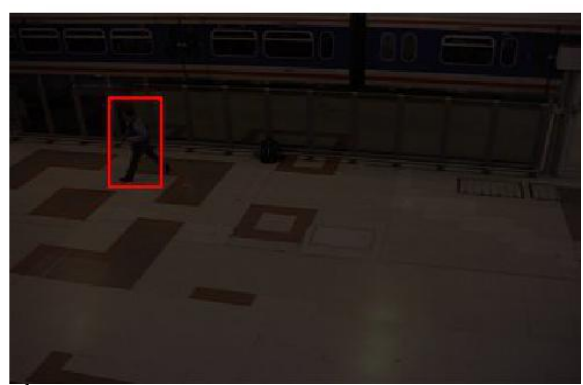

(d) $20^{\text {th }}$ Frame

Figure 11: Sequence from PETS 2006 database, tracking with conventional MST 


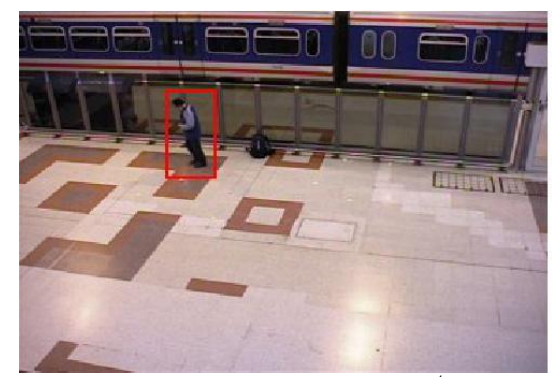

(a) $1^{\text {st }}$ Frame

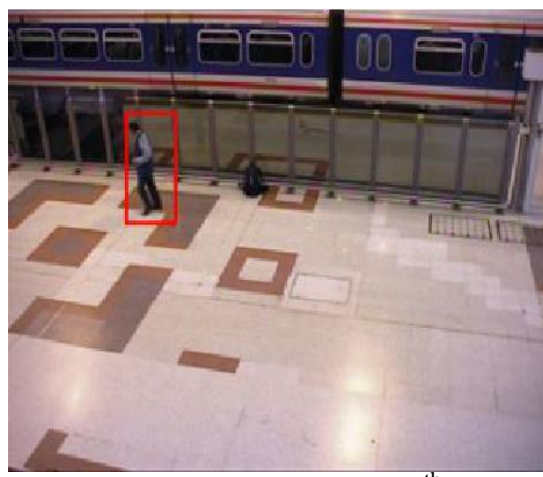

(c) $15^{\text {th }}$ Frame

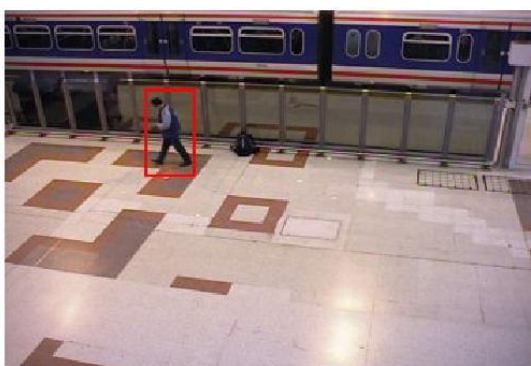

(b) $4^{\text {th }}$ Frame

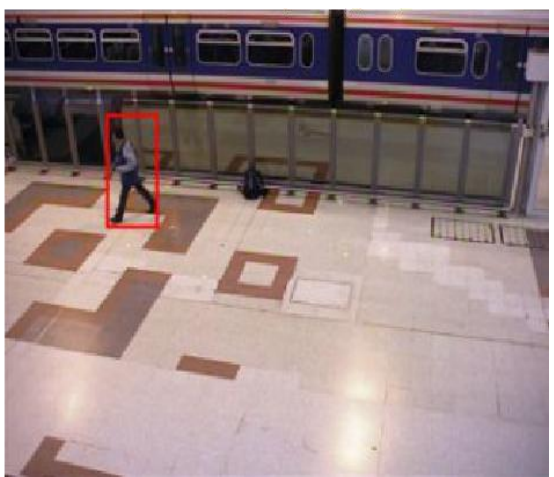

(d) $20^{\text {th }}$ Frame

Figure 12: Sequence from PETS 2006 database, tracking with proposed approach

\section{Conclusion}

A low complex and faster based on illumination invariant object tracking and detection scheme are proposed in this paper, it is observed that the proposed approach is providing accurate results when tested both in real time and standard data set videos under varying illumination. This work is providing an improvement of $14 \%$ in detection ratio when compared with the conventional MST approach with respect to ground templates. This work may be further extended to be implemented for the recognition of multiple objects and unattended object tracking.

\section{References}

[1] V. M. P. Comaniciu, D and Ramesh, "Real-time tracking of non-rigid objects using mean shift", In Computer Vision and Pattern Recognition, 2000, Proc. IEEE Conf. on, 2000

[2] Alan J Lipton, Hironobu Fujiyoshi, and Raju S Patil, "Moving target classification and tracking from a real-time video", In Applications of Computer Vision, 1998. WACV'98 Proceedings, Fourth IEEE Workshop on, pages 8-14, IEEE, 1998

[3] Ya Liu, Haizhou Ai, and Guang-you Xu, "Moving object detection and tracking based on background subtraction", In Multispectral Image Processing and Pattern Recognition, pages 62-66. International Society for Optics and Photonics, 2001

[4] T. Celik and T. Tjahjadi, "Automatic image equalization and contrast enhancement using Gaussian mixture modeling", Image Processing, IEEE Transactions, Jan- 2012

[5] H. Xu, G. Zhai, X. Wu and X. Yang, "Generalized Equalization Model for Image Enhancement," in IEEE Transactions on Multimedia, vol. 16, no. 1, pp. 68-82, Jan. 2014.

[6] G. Phadke and R. Velmurgan, "Illumination invariant Mean-shift tracking," 2013 IEEE Workshop on Applications of Computer Vision (WACV), Tampa, FL, 2013.

[7] http://www.cvg.reading.ac.uk/PETS2006/data.html

[8] http://www.dahuasecurity.com/

[9] J. Wang and Y. Yagi, "Integrating color and shape texture features for adaptive real-time object tracking", Image Processing, IEEE Transactions, Feb 2008.

[10] K. Warhade, S. Merchant, and U. Desai, "Avoiding false positive due to flashlights in shot detection using illumination suppression algorithm", IET Conference Publications, 2008. 